\title{
Salicylic Acid and Jasmonic Acid Are Essential for Systemic Resistance Against Tobacco mosaic virus in Nicotiana benthamiana
}

\author{
Feng Zhu, ${ }^{1}$ De-Hui Xi, ${ }^{1}$ Shu Yuan, ${ }^{1}$ Fei Xu, ${ }^{1}$ Da-Wei Zhang, ${ }^{1}$ and Hong-Hui Lin ${ }^{1,2}$ \\ ${ }^{1}$ Ministry of Education Key Laboratory for Bio-Resource and Eco-Environment, College of Life Science, and ${ }^{2}$ State Key \\ Laboratory of Hydraulics and Mountain River Engineering, Sichuan University, Chengdu 610065, China
}

Submitted 21 November 2013. Accepted 14 January 2014.

Systemic resistance is induced by pathogens and confers protection against a broad range of pathogens. Recent studies have indicated that salicylic acid (SA) derivative methyl salicylate (MeSA) serves as a long-distance phloemmobile systemic resistance signal in tobacco, Arabidopsis, and potato. However, other experiments indicate that jasmonic acid (JA) is a critical mobile signal. Here, we present evidence suggesting both MeSA and methyl jasmonate (MeJA) are essential for systemic resistance against Tobacco mosaic virus (TMV), possibly acting as the initiating signals for systemic resistance. Foliar application of JA followed by SA triggered the strongest systemic resistance against TMV. Furthermore, we use a virus-induced gene-silencingbased genetics approach to investigate the function of JA and SA biosynthesis or signaling genes in systemic response against TMV infection. Silencing of SA or JA biosynthetic and signaling genes in Nicotiana benthamiana plants increased susceptibility to TMV. Genetic experiments also proved the irreplaceable roles of MeSA and MeJA in systemic resistance response. Systemic resistance was compromised when SA methyl transferase or JA carboxyl methyltransferase, which are required for MeSA and MeJA formation, respectively, were silenced. Moreover, high-performance liquid chromatography-mass spectrometry analysis indicated that JA and MeJA accumulated in phloem exudates of leaves at early stages and $\mathrm{SA}$ and MeSA accumulated at later stages, after TMV infection. Our data also indicated that JA and MeJA could regulate MeSA and SA production. Taken together, our results demonstrate that (Me)JA and (Me)SA are required for systemic resistance response against TMV.

Because plants are constantly attacked by pathogens, including bacteria, fungi, oomycetes, and viruses, they have evolved extraordinarily complex mechanisms to defend themselves (Dangl and Jones 2001). Plants respond to biotic challenge via inducible basal defense networks activated through recognition

F. Zhu, D.-H. Xi, and S. Yuan contributed equally to this work.

Corresponding author: H.-H Lin; Telephone: +1 86-28-85415389; Fax: +1 86-28-85412571; E-mail: hhlin@scu.edu.cn

* The $e$-Xtra logo stands for "electronic extra" and indicates that seven supplementary figures, two supplementary tables, and three supplementary methods are published online and that the figures appear in color online.

(C) 2014 The American Phytopathological Society and response to conserved pathogen-associated molecular patterns (PAMPs) (Halim et al. 2009). This PAMP-triggered immunity is very effective, because most plants are resistant to the majority of pathogens (Jones and Dangl 2006). However, pathogens have acquired effectors to counteract basal resistance, leading to the successful colonization of the host plant. Resistance $(R)$ genes in the plant evolve to recognize effectors or their activities that initiate effector-triggered immunity. For example, the tobacco resistance gene $N$ is a typical $R$ gene, and the $\mathrm{N}$ protein specifically recognizes the $50-\mathrm{kDa}$ helicase domain of the Tobacco mosaic virus (TMV) replicase protein (Erickson et al. 1999). Effector-triggered immunity resulting in hypersensitive response (HR) is frequently accompanied by subsequent systemic immunity responses such as systemic acquired resistance (SAR). Plants are able to express induced defense at sites remotely located from the initial infection, resulting in enhanced systemic resistance to subsequent pathogen encounter. SAR and induced systemic resistance (ISR) are two types of systemic defense responses (Durrant and Dong 2004; Grant and Lamb 2006). SAR is a form of inducible resistance that occurs in systemic tissues after a pathogen triggers a localized HR that results in necrotic lesions on the inoculated leaf (Durrant and Dong 2004). SAR responses are associated with the accumulation of salicylic acid (SA) and the transcriptional upregulation of a number of genes, including those encoding pathogenesis-related (PR) proteins such as $P R 1, P R 2$, and $P R 5$ in both local and systemic tissues (Durrant and Dong 2004). In contrast, ISR is dependent on jasmonic acid (JA) and ethylene (ET) pathways and is not associated with PR expression (Pieterse et al. 1998).

The phytohormones SA, JA, and ET are known to participate in defense responses in plants (van Wees et al. 2000). The signaling pathways influence each other through a complex network of synergistic and antagonistic interactions (Koornneef and Pieterse 2008). In many cases, ET has been shown to act as a modulator of plant responses to either SA or JA (Adie et al. 2007). Kwon and associates (2009) showed that Arabidopsis GDSL LIPASE-LIKE 1 (GLIP1) can generate and propagate a systemic signal that is required for ET-mediated systemic resistance. Signaling is a central part of systemic resistance that requires some potential mobile signals (Chanda et al. 2011; Durrant and Dong 2004; Shah 2009). It is believed that a signal is generated at the primary infection site and is subsequently translocated to distal plant tissues to activate defense mechanisms (Grant and Lamb 2006). Several candidates, including methyl salicylate (MeSA), jasmonates, azelaic acid, abietane diterpenoid dehydroabietinal (DA), pipecolic acid, glycerol-3phosphate $(\mathrm{G} 3 \mathrm{P})$, and green leaf volatiles have been suggested 
as the signaling molecules that induce SAR (Chanda et al. 2011; Fu and Dong 2013; Kachroo and Robin 2013; Návarová et al. 2012; Shah 2009; Shah and Zeier 2013). SA plays an important role in establishing and maintaining SAR (Vlot et al. 2008a). Direct evidence for the role of SA in plant defense comes from the identification and characterization of an Arabidopsis isochorismate synthase (ICS) mutant (sid2-2) that is defective in SA biosynthesis (Wildermuth et al. 2001). Silencing of ICS in Nicotiana benthamiana enhanced Agrobacterium infection (Anand et al. 2008). Exogenous application of SA or its functional analogs, such as 2,6-dichloroisonicotonic acid and benzo-(1,2,3)-thiadiazole-7-carbothioic acid S-methyl ester (BTH), induces SAR in plants, resulting in resistance to certain pathogens (Achuo et al. 2004). The fact that SA accumulates in the phloem and is required to activate SAR led to the proposal of SA as the mobile signal (Yalpani et al. 1991). However, grafting experiments demonstrate that SA is not the systemic signal (Vernooij et al. 1994). Park and associates (2007) found that SA is converted to MeSA, which is synthesized from SA by an SA methyl transferase (SAMT) and can act as a mobile signal for plant resistance to TMV. Structural and biochemical analyses indicate that salicylic acid-binding protein 2 (SABP2) has strong esterase activity toward the substrate MeSA and converts MeSA to SA, and that SA is a potent feedback inhibitor of SABP2 (Forouhar et al. 2005; Kumar and Klessig 2003).

However, there is evidence that the jasmonates 12-oxo-phytodienoic acid (OPDA), JA, and methyl jasmonic acid (MeJA) act as active systemic signaling molecules (Cheong and Choi 2003; Wasternack 2007). Truman and associates (2007) found that JA but not SA rapidly accumulates in phloem exudates of leaves challenged with an avirulent bacterial strain of Pseudomonas syringae pv. tomato and suggested that JA may be the key signaling molecule to systemic immunity, although JA plays an important role in defenses against necrotrophic pathogens and insect attack. JA regulates the expression of a series of genes, including $P R-3$, vegetative storage protein 2 (VSP2), thionin 2.1 (THI2.1), and plant defensin 1.2 (PDF1.2), which activate defense responses and enable plants to acquire resistance (Liu et al. 2005; Memelink 2009; Wu et al. 2008). It has also been established that a highly active jasmonate is perceived by the receptor CORONATINE INSENSITIVE1 (COI1) (Katsir et al. 2008). However, Attaran and associates (2009) indicated that neither MeSA nor JA is essential for systemic immunity, emphasizing the crucial role of SA in Arabidopsis infected with $P$. syringae. The nature of the mobile signal and remotely activated networks responsible for establishing SAR remain unclear.

In this report, we investigated the role of SA and JA in systemic resistance responses in $N$. benthamiana. We use a virusinduced gene-silencing (VIGS)-based genetics approach (Rojas et al. 2012; Zhu et al. 2014) to investigate the function of JA and SA biosynthesis or signaling genes in systemic resistance response against TMV infection.

\section{RESULTS}

Foliar hormone applications trigger systemic resistance in $N$. benthamiana plants against TMV.

We tested control and treated $N$. benthamiana plants for their resistance against infection using green fluorescent protein (GFP)-tagged TMV, a recombinant TMV that is infectious (Liu et al. 2002b). Wild-type (WT) N. benthamiana treated with water was used as control. Tobacco leaves were treated at one site on the primary leaf with plant hormones, then inoculated with TMV-GFP on the secondary (systemic) leaves. Systemic symptoms occurred mainly in the four leaves located above the inoculated leaves but also reached leaves further up. Virus accumulation was confirmed by direct observation of GFP fluorescence (Fig. 1A), as well as by quantitative realtime polymerase chain reaction (PCR) analysis of viral RNA (Fig. 1B and C). N. benthamiana plants treated with JA followed by SA appeared to have the weakest GFP fluorescence in the inoculated leaves and systemic leaves (Fig. 1A) at 6 and 12 days postinoculation (dpi), respectively. The conclusion is consistent with real-time PCR analysis of viral RNA accumulation (Fig. 1B and C). Plants treated with JA followed by SA have the lowest levels of viral RNA in comparison with control (Fig. 1B and C). Exogenous application of SA, JA, SA + JA, and $\mathrm{SA} \rightarrow \mathrm{JA}$ also yielded a significant reduction in the GFP fluorescence (Fig. 1A) and the levels of viral RNA per plant (Fig. 1B and C).

Taken together, we conclude that SA and JA in combination are likely strong activators of systemically induced defense in tobacco leaves against TMV. Moreover, the sequential application of JA followed by SA appears to enhance $N$. benthamiana immunity to TMV.

\section{Silencing of SA or JA biosynthetic and signaling genes in $N$. benthamiana plants increases susceptibility to TMV.}

To further investigate the role of SA and JA in limiting TMV infectivity in $N$. benthamiana plants, we used a Tobacco rattle virus (TRV)-based VIGS system as a rapid genetics tool (Anand et al. 2008; Liu et al. 2002a and b) to silence SA or JA biosynthetic and signaling genes in $N$. benthamiana plants and examined the functions in TMV infections. We targeted the following genes: NbICS1, homolog of Arabidopsis thaliana, which is involved in SA biosynthesis (Anand et al. 2008; Wildermuth et al. 2001); NbSABP2 (SA-binding protein 2), which converts inactive MeSA to active SA (Kumar and Klessig 2003); NbSAMT (SA methyl transferase), which catalyzes SA into MeSA (Kumar and Klessig 2003); NbNPR1 (nonexpresser of $P R$ gene 1), which is involved in SA signaling (Cao et al. 1997); NbOPR3 (12-oxo-phytodienoic acid reductase), which is involved in JA biosynthesis (Breithaupt et al. 2006); NbJMT (jasmonic acid carboxyl methyltransferase), which catalyzes JA into MeJA (Seo et al. 2001); and NbCOI1 (coronatine insensitive 1), which is involved in JA signaling (Xie et al. 1998). These genes were isolated by reverse-transcription (RT)-PCR and cloned into the vector for TRV-mediated VIGS.

We first assessed the gene silencing efficiency of TRV-VIGS clones by suppressing the expression of the phytoene desaturase $(P D S)$ gene in $N$. benthamiana. Silencing of $P D S$ leads to the inhibition of carotenoid synthesis, causing the plants to exhibit a photo-bleached phenotype (Supplementary Fig. S1). All gene-silenced plants showed normal growth compared with the control TRV-infected plant (Supplementary Fig. S2). The downregulation of the NbPDS, NbICS1, NbNPR1, NbSABP2, NbSAMT, NbOPR3, NbJMT, and NbCOI1 genes in silenced plants was confirmed by RT-PCR after 12 days of infiltration (Supplementary Fig. S3).

Silenced plants were infected with TMV-GFP and monitored for viral replication and spread for at least 2 weeks. We found that the NbICS1-, NbNPR1-, NbSABP2-, NbSAMT-, NbOPR3-, NbJMT-, and NbCOI1-silenced plants accumulated more virus compared with the control (Fig. 2A). TMV-GFP moved into the veins of inoculated leaves and spread into the upper uninoculated leaves, and these silenced plants had stronger GFP fluorescence in the noninoculated upper leaves. Quantitative real-time PCR (Fig. 2B) and Western blotting (Fig. 2C) analyses were used to quantify virus accumulation in these silenced plants. Higher viral accumulation was observed in the systemic leaves of these silenced plants as compared 
with control plants (Fig. 2B). These silenced plants showed 1.9- to 2.5-fold increase in TMV mRNA levels at 15 dpi in comparison with control plants (Fig. 2B). Furthermore, the levels of TMV coat protein accumulations were also higher in these silenced plants in comparison with basal expression levels (Fig. 2C). This difference was more obvious in NbSABP2-, NbSAMT-, NbNPR1-, and NbOPR3-silenced plants. Overall, our results show that disturbance of JA- and SA-mediated defense pathways led to hypersusceptibility to TMV.

\section{Expression of defense-related genes in SA}

or JA biosynthetic and signaling gene-silenced plants.

To examine whether the expression of JA and SA defense marker genes was differentially regulated in SA or JA biosynthetic and signaling gene-silenced plants infected with TMVGFP, the expression of $P R 1, P R 2$, and $P R 5$ (SA marker genes) as well as $P D F 1.2$ and $P R 3$ (JA marker genes) was investigated by quantitative real-time PCR. The expression of $P R 1$, $P R 2$, and $P R 5$ was not significantly altered in control plants at $1 \mathrm{dpi}$ in comparison with basal expression levels, and increased significantly at $5 \mathrm{dpi}$ (Fig. $3 \mathrm{~A}$ to $\mathrm{C}$ ). The expression of $P R 1$, $P R 2$, and PR5 was not upregulated in NbICS1-, NbSABP2-, $N b S A M T$-, and NbNPR1-silenced plants in which the SA pathway was affected (Fig. $3 \mathrm{~A}$ to $\mathrm{C}$ ). On the other hand, PDF1.2 and $P R 3$ mRNAs were significantly upregulated (3.1- and 3.3fold, respectively) in WT plants infected with TMV-GFP (TRV:00, control) at $1 \mathrm{dpi}$ in comparison with leaves from noninfected plants, and their expression was not upregulated in NbOPR3-, NbJMT-, and NbCOI1-silenced plants in which the JA pathway was affected compared with the control (TRV:00) plants (Fig. 3D and E). The expression of PDF1.2 and PR3 decreased significantly (0.4- and 0.37 -fold, respectively) in control plants at 5 compared with $1 \mathrm{dpi}$ (Fig. 3D and E). These results suggest that JA-mediated defense genes are first activated, after which SA-mediated defense genes are activated in $N$. benthamiana plants infected with TMV-GFP.

\section{NahG-transgenic $N$. benthamiana plants with silenced JA biosynthetic and signaling genes are hypersusceptible to TMV.}

Because we have shown that both JA and SA pathways are required for systemic response to TMV (Fig. 2), we decided to examine the relationship between these two hormonal pathways. The SA-dependent pathway has been analyzed using transgenic plants expressing the salicylate hydroxylase $(N a h G)$ gene, which degrades SA to catechol (Gaffney et al. 1993). NahG-expressing tomato (Solanum lycopersicum) plants show an increased susceptibility to viral and bacterial pathogens, which is correlated with a reduction in expression of $P R 1$ (Gaffney et al. 1993). The accumulation of $N a h G$ transcripts in NahG-transgenic N. benthamiana plants was confirmed by RT-PCR (Supplementary Fig. $\mathrm{S} 4 \mathrm{~A}$ ). The endogenous levels of SA were much lower in NahG plants than in WT plants. We found that $N a h G$-expressing plants were more susceptible to TMV-GFP infection, as indicated by the increased green fluorescence (Fig. 4A) and the increased levels of viral RNA (Fig. 4B) compared with the WT N. benthamiana plants. To silence JA biosynthetic and signaling genes in $N a h G$-transgenic $N$. benthamiana plants, silencing vectors TRV:NbOPR3, TRV:NbJMT, and TRV:NbCOII were introduced into $N a h G$ plants. The empty vector TRV:00 was introduced into $N a h G$ plants as control. Plants treated with the three silencing constructs showed normal growth compared with the control
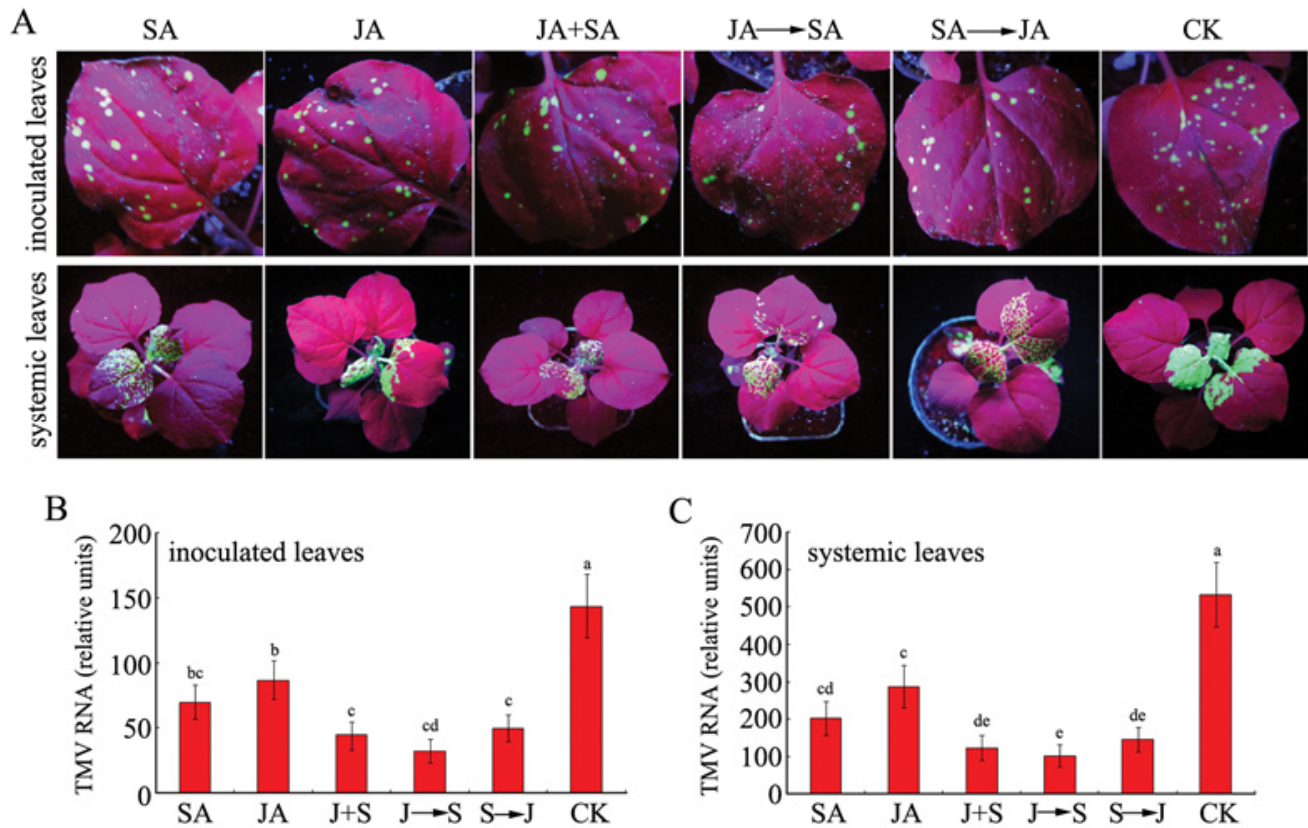

Fig. 1. Salicylic acid (SA) and jasmonic acid (JA) are involved in systemic resistance response against TMV. A, Effects of foliar application of plant hormones (SA, JA, JA+SA, JA $\rightarrow \mathrm{SA}, \mathrm{SA} \rightarrow \mathrm{JA}$ ) on systemic plant defense against TMV-green fluorescent protein (GFP) infection in Nicotiana benthamiana plants. These plant hormones were divided into 2-day treatments. "SA" indicates half of SA pretreatment in the first day and half of SA pretreatment in the second day; "JA" indicates half of JA pretreatment in the first day and half of JA pretreatment in the second day; "J + S" means half of JA + SA pretreatment in the first day and half of JA+SA pretreatment in the second day; "J $\rightarrow \mathrm{S}$ " indicates total of JA pretreatment in the first day and total of SA pretreatment in the second day; "S $\rightarrow \mathrm{J}$ " indicates total of SA pretreatment in the first day and total of JA pretreatment in the second day; "CK" indicates half of distilled water pretreatment in the first day and half of distilled water pretreatment in the second day. In "J + S," "J $\rightarrow$ S," and "S $\rightarrow J$," each JA and SA amount was half of "SA" and "JA" and the total amount of "J $+\mathrm{S}$," "J $\rightarrow \mathrm{S}$," and "S $\rightarrow \mathrm{J}$ " was same as "SA" and "JA." N. benthamiana plants were treated at one site on the primary leaf with plant hormones, then inoculated with TMV-GFP on the secondary (systemic) leaves. TMV-GFP spread in the inoculated leaves and systemic leaves were photographed at 6 and 12 days postinoculation (dpi), respectively. Experiments were repeated three times, with similar results. B and $\mathbf{C}$, Quantitative real-time polymerase chain reaction analysis of TMV accumulation levels in the inoculated leaves and the systemic leaves collected at 6 and 12 dpi, respectively. Bars represent mean and standard deviation of values obtained from three biological replicates per genotype and time point. Actin was used as the internal reference gene. Significant differences $(P<0.05)$ are denoted by different lowercase letters. 
TRV-infected plant after 12 days of infiltration. Extensive green fluorescence and more severe symptoms were observed in the systemic leaves in $N b O P R 3-, N b J M T$-, and $N b C O I 1$-silenced plants than in control plants (NahG + TRV:00), which displayed a few fluorescent areas (Fig. 4A). Furthermore, blocking JA bio-

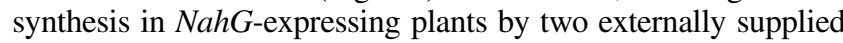
lipoxygenase (LOX) inhibitors, salicylhydroxamic acid (SHAM), and diethyldithiocarbamic acid (DIECA) (Nahar et al. 2011) significantly increased the plants' susceptibility toward TMV-GFP (Fig. 4A). NahG-expressing plants treated with SHAM or DIECA showed stronger symptoms and fluorescence in newly emerged leaves (Fig. 4A). To corroborate our fluorescence assay, quantitative real-time PCR was performed to detect viral mRNA levels. Viral RNA significantly increased in NbOPR3-, NbJMT-, and NbCOIl-silenced NahG-transgenic plants at 12 dpi compared with WT plants (Fig. 4B). These silenced plants showed 3.3- to 3.8-fold increase of TMV mRNA levels at 12 dpi in comparison with WT plants (Fig. 4B). Furthermore, similar results were observed in $N a h G$-expressing plants treated with SHAM or DIECA (Fig. 4B). Based on these results, we conclude that SA and JA are both required for plant defenses against TMV infection in $N$. benthamiana plants. It's also important to note that removal of SA by NahG appears to have a major effect on TMV infection, and JA acts to modulate SA activity (Fig. 4). The results can be best explained in a model in which JA and SA function in the same pathway to confer resistance to TMV.

Foliar hormone applications could trigger systemic resistance in plants deficient in both SA and JA.

To further support the idea that both JA and SA are required for systemic resistance against TMV, we performed the following experiments. NbOPR3- and NbJMT-silenced $N a h G$-transgenic plants were treated with five hormone combinations, after which TMV-GFP was inoculated. BTH, which mimics $\mathrm{SA}$ in inducing disease resistance in various hostpathogen systems but cannot be catabolized by NahG (Achuo et al. 2004), was used in place of SA. BTH did not induce SA accumulation in $N$. benthamiana plants. $N b O P R 3$ - and $N b J M T$-silenced $N a h G$-transgenic plants treated with JA fol-

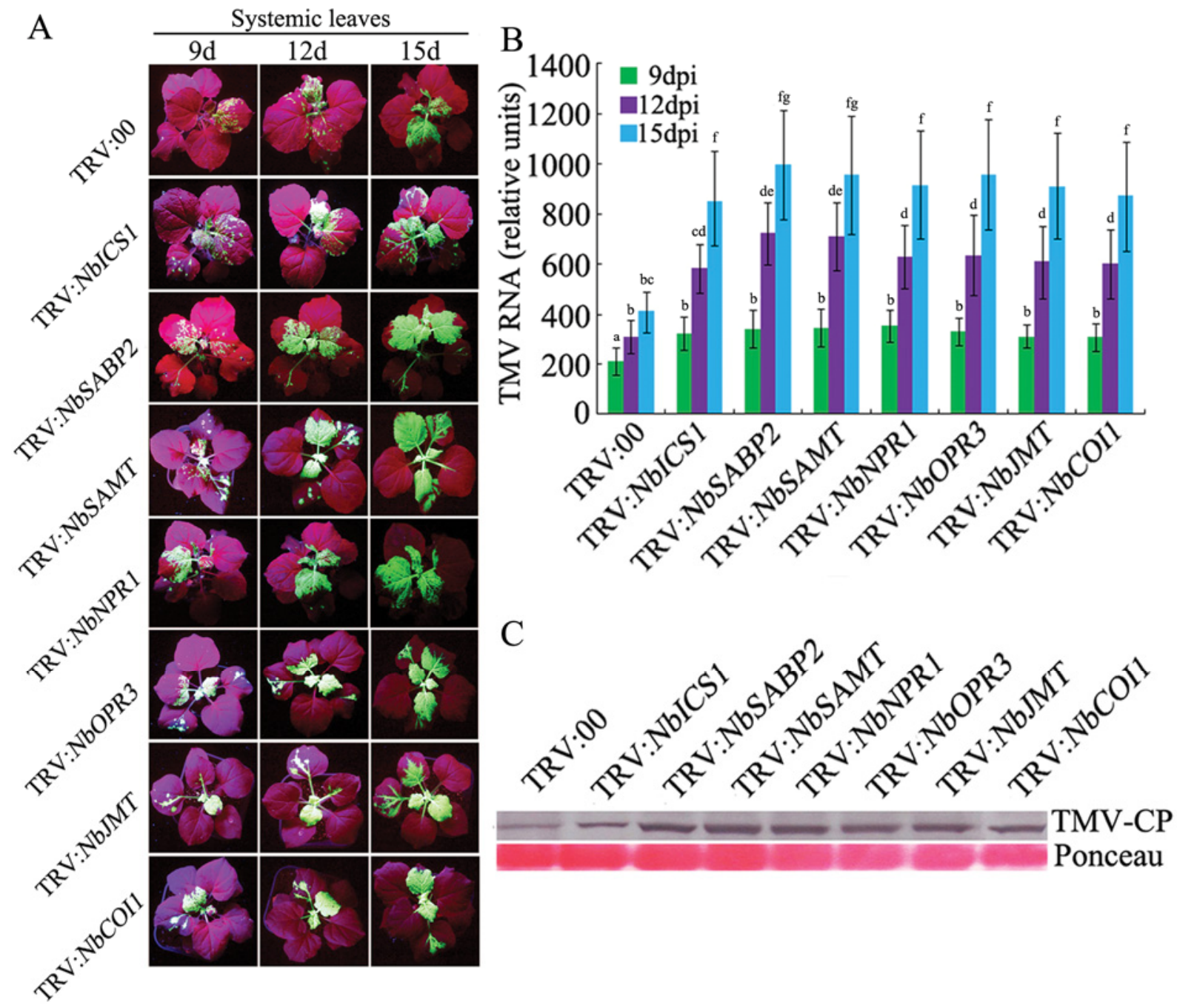

Fig. 2. Silencing of salicylic acid (SA) or jasmonic acid (JA) biosynthetic and signaling genes increases susceptibility to TMV. A, Analysis of green fluorescent protein (GFP) fluorescence in the gene-silenced plants. The TMV-GFP spread assay was performed on NbICS-, NbSABP2-, NbSAMT-, NbNPRI-, NbOPR3-, NbJMT-, and NbCOI1-silenced and control (TRV:00) Nicotiana benthamiana seedlings. Photographs were taken from the systemic leaves 9, 12, and 15 days postinoculation (dpi). Experiments were repeated three times with similar results. B, Quantitative real-time polymerase chain reaction analysis of TMV mRNA accumulation levels in the systemic leaves 9, 12, and 15 dpi. Bars represent mean and standard deviation of values obtained from three biological replicates per genotype and time point. Actin was used as the internal reference gene. Different lowercase letters indicate significant differences $(P<$ 0.05). C, Western blotting analysis of coat protein accumulation of TMV in the gene-silenced plants infected with TMV-GFP 12 dpi. Approximately $3 \mu \mathrm{g}$ of protein from each sample was loaded on gels. Rubisco proteins were used as loading controls and were stained by Ponceau S. Experiments were repeated three times with similar results. 
lowed by BTH appeared to have the weakest GFP fluorescence in the systemic leaves (Fig. 5A and B), with the extraordinarily low levels of viral RNA (Fig. 5C and D). These plants treated with JA followed by BTH had significantly reduced TMV mRNA levels. Exogenous application of BTH, JA, BTH + JA, and $\mathrm{BTH} \rightarrow \mathrm{JA}$ also yielded a smaller reduction in the GFP fluorescence (Fig. 5A and B) and levels of viral RNA per plant (Fig. 5C and D). These data further support the idea that both SA and JA signaling are essential for systemic resistance in $N$. benthamiana.
Accumulations of JA followed by SA

in $N$. benthamiana plants infected with TMV.

To further confirm that MeSA and jasmonate signaling are essential for systemic resistance, we conducted phytohormone analyses in WT; TRV:00; NbSABP2-, NbSAMT-, NbOPR3-, and $N b J M T$-silenced $N$. benthamiana plants; NahG-transgenic plants; and NbOPR3-silenced $N a h G$-transgenic plants infected with TMV-GFP. Endogenous levels of SA, MeSA, JA, and MeJA were measured using high-performance liquid chromatography (HPLC) electrospray ionization tandem mass spec-
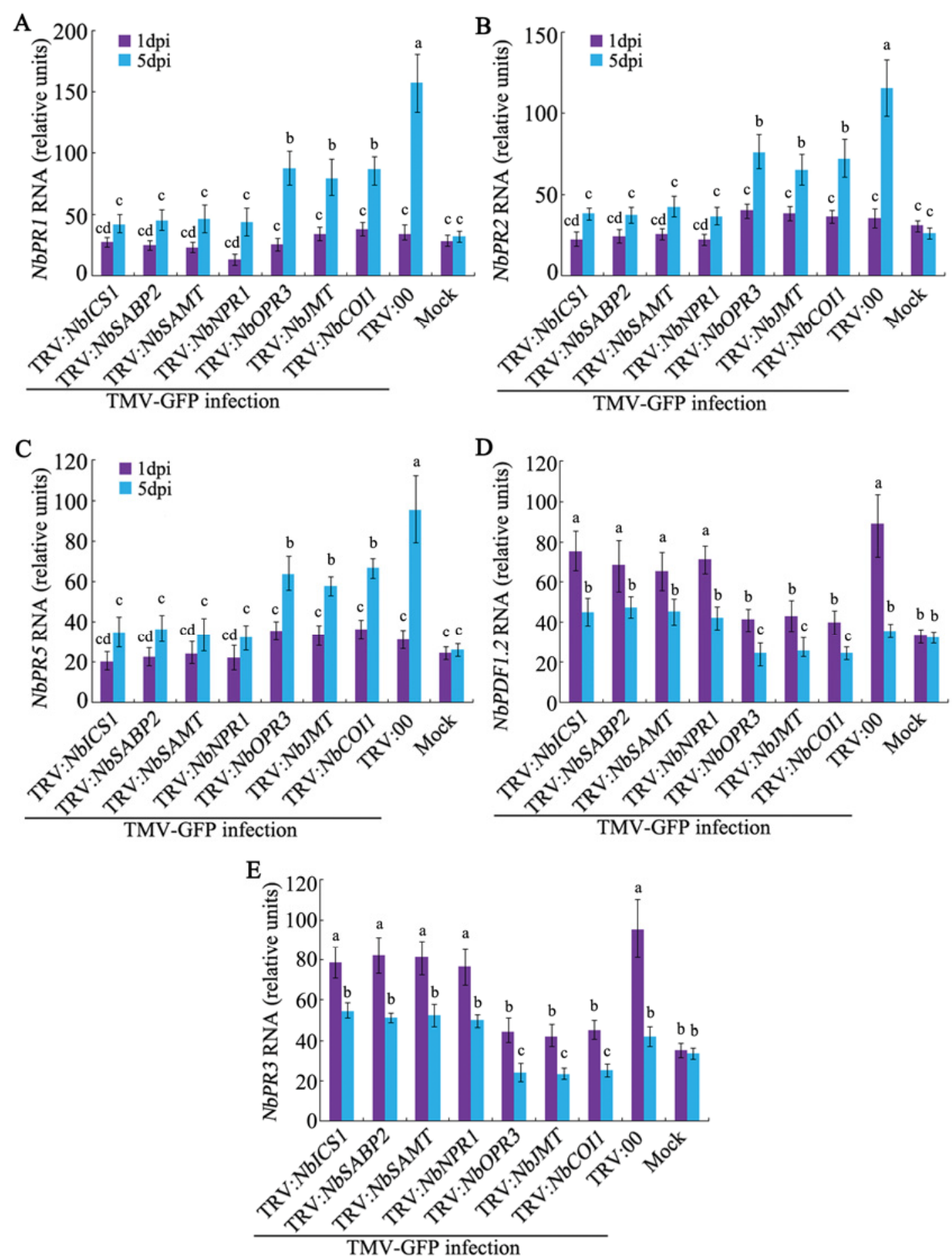

Fig. 3. Quantitative real-time polymerase chain reaction analysis expressions of defense-related genes in the gene-silenced plants infected with TMV-green fluorescent protein (GFP) 1 or 5 days postinoculation (dpi). A to C, Expression of defense-related genes associated with the salicylic-acid-mediated defense pathway. D and E, Expression of defense-related genes associated with the jasmonic-acid-mediated defense pathway. "Mock" means seedlings not infected with TMV-GFP. Bars represent mean and standard deviation of values obtained from three biological replicates per genotype and time point. Actin was used as the internal reference gene. Significant differences $(P<0.05)$ are denoted by different lowercase letters. 
trometry (Pan et al. 2010). We found that endogenous levels of JA (Fig. 6A, C, and E; Supplementary Fig. S5A, C, and E) and MeJA (Fig. 7A, C, and E; Supplementary Fig. S6A, C, and E) increased rapidly in inoculated leaves, phloem exudates, and systemic leaves of WT plants, TRV:00, NbSABP2- and $N b S A M T$-silenced plants, and $N a h G$-transgenic plants from 6 to $48 \mathrm{~h}$ postinfection (hpi). As expected, JA and MeJA levels were not significantly different in NahG-transgenic plants and
NbSAMT-silenced plants compared with WT and TRV:00, indicating that SA does not affect JA production. JA and MeJA reached the highest levels at $48 \mathrm{hpi}$ and started to decrease from 72 to 120 hpi (Figs. 6 and 7). The endogenous levels of JA and MeJA were much less in NbOPR3-silenced plants and NbOPR3-silenced NahG-transgenic plants than in WT plants (Figs. 6 and 7) and JA levels were slightly decreased in NbJMTsilenced plants (Fig. 6A, C, and E). On the other hand, endoge-

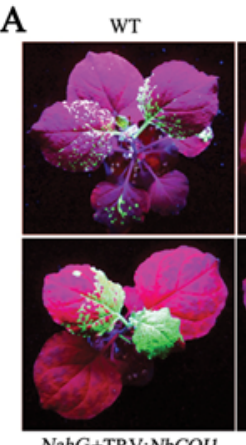

$\mathrm{NahG+TRV:NbCOII}$
TRV:00

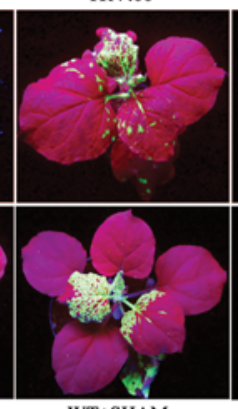

WT+SHAM

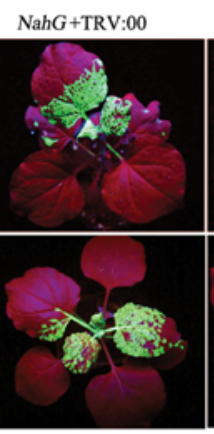

WT+DIECA

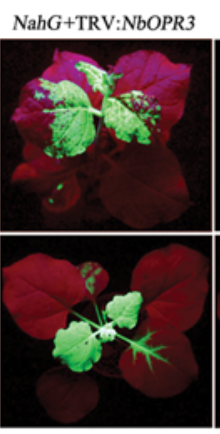

NahG + SHAM

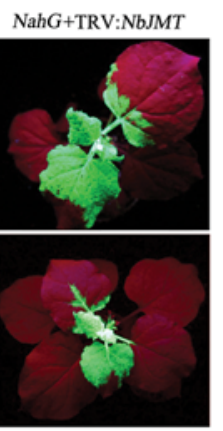

NahG + DIECA

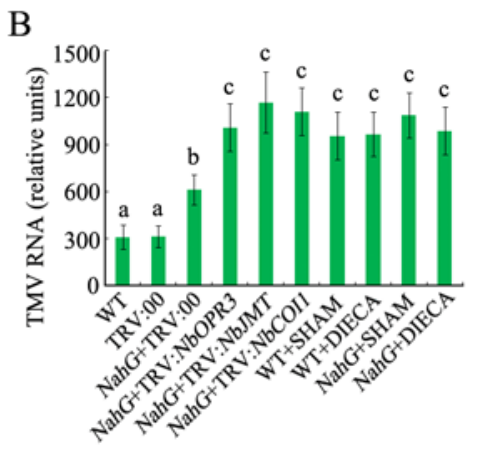

Fig. 4. Nicotiana benthamiana plants deficient in both salicylic acid (SA) and jasmonic acid (JA) are hypersusceptible to TMV. A, $N a h G$-transgenic $N$. benthamiana plants that silenced JA biosynthetic and signaling genes or were treated with JA biosynthesis inhibitor are hypersusceptible to TMV. TMVgreen fluorescent protein (GFP) spread in the systemic leaves photographed at 12 days postinoculation (dpi). Experiments were repeated three times with similar results. WT = wild type. B, Quantitative real-time polymerase chain reaction analysis of TMV mRNA accumulation levels at $12 \mathrm{dpi}$. Bars represent mean and standard deviation of values obtained from three biological replicates per genotype. Actin was used as the internal reference gene. Different lowercase letters indicate significant differences $(P<0.05)$

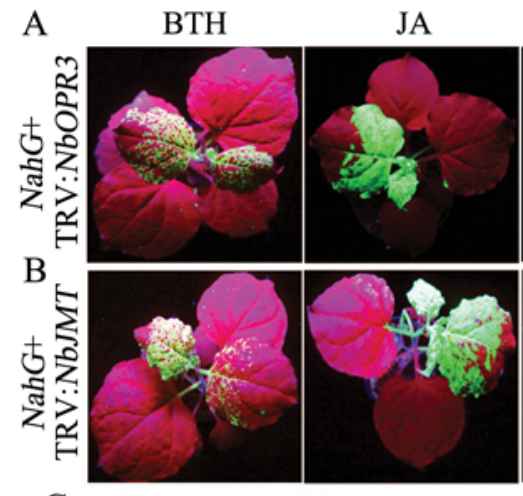

C

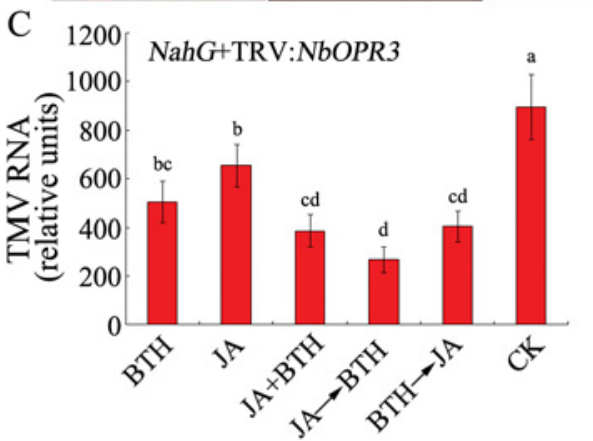

$\mathrm{JA}+\mathrm{BTH}$
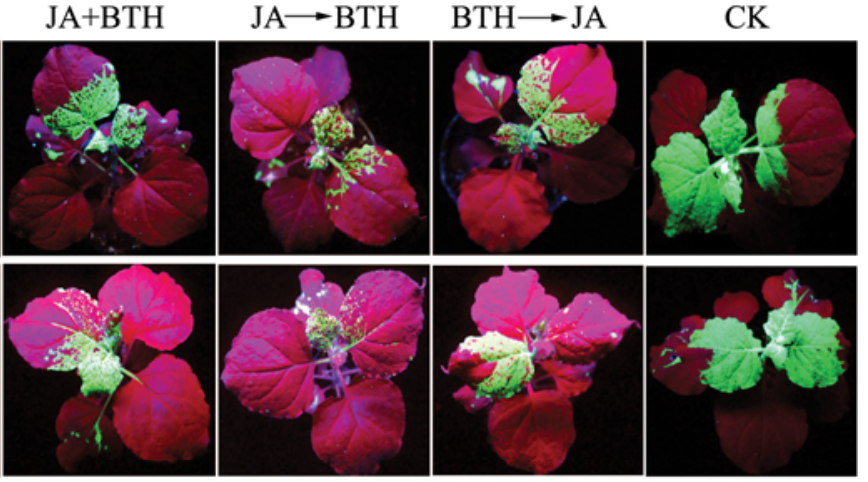

D 1200

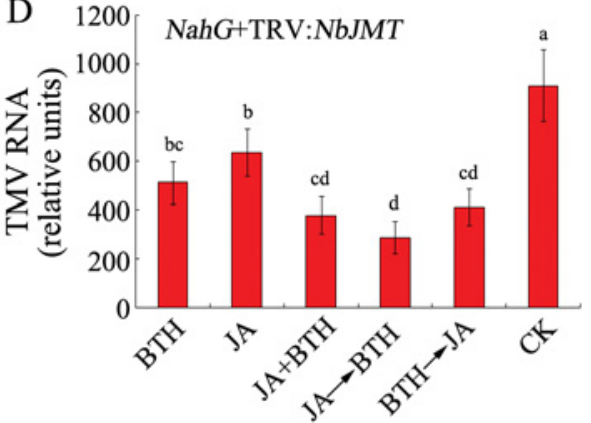

Fig. 5. Foliar hormone applications could trigger systemic resistance in plants deficient in both salicylic acid (SA) and jasmonic acid (JA). A and B, Effects of foliar application of plant hormones or benzo-(1,2,3)-thiadiazole-7-carbothioic acid $S$-methyl ester (BTH) on systemic plant defense against TMV-green fluorescent protein (GFP) infection in NbOPR3- and NbJMT-silenced $N a h G$-transgenic plants. TMV-GFP spread in the systemic leaves photographed at 12 days postinoculation (dpi). These plant hormones were divided into 2-day treatments. "BTH" indicates half of BTH pretreatment in the first day and half of BTH pretreatment in the second day; "JA" indicates half of JA pretreatment in the first day and half of JA pretreatment in the second day; "JA + BTH" means half of JA + BTH pretreatment in the first day and half of JA + BTH pretreatment in the second day; "JA $\rightarrow$ BTH" indicates total of JA pretreatment in the first day and total of BTH pretreatment in the second day; "BTH $\rightarrow$ JA" indicates total of BTH pretreatment in the first day and total of JA pretreatment in the second day; "CK" indicates half of distilled water pretreatment in the first day and half of distilled water pretreatment in the second day. In "JA + BTH," "JA $\rightarrow$ BTH," and "BTH $\rightarrow$ JA," each JA and BTH amount was half of "BTH" and "JA" and the total amount of "JA + BTH," "JA $\rightarrow$ BTH," and "BTH $\rightarrow$ JA" was same as "BTH" and "JA." NbOPR3- and NbJMT-silenced NahG-transgenic plants were treated at one site on the primary leaf with plant hormones, then inoculated with TMV-GFP on the secondary (systemic) leaves. Experiments were repeated three times with similar results. C and D, Quantitative real-time polymerase chain reaction analysis of TMV mRNA accumulation levels at 12 dpi. Bars represent mean and standard deviation of values obtained from three biological replicates. Actin was used as the internal reference gene. Significant differences $(P<0.05)$ are denoted by different lowercase letters. 
nous levels of MeJA were significantly reduced in $\mathrm{NbJMT}$ silenced plants (Fig. 7A, C, and E). In addition, no significant difference was observed for the JA and MeJA in NbOPR3silenced plants and $N b O P R 3$-silenced $N a h G$-transgenic plants from 0 to 120 hpi (Figs. 6 and 7).

In contrast, SA and MeSA have different accumulation patterns. There were not many changes for endogenous levels of SA in inoculated leaves of TRV:00 and WT plants at the early stage (from 0 to $48 \mathrm{hpi}$ ). However, SA levels started to increase in inoculated leaves, phloem, and systemic leaves of TRV:00 and WT plants at later period (from 48 to $120 \mathrm{hpi}$ ) (Fig. 6B, D, and F. MeSA levels in TRV:00 and WT plants increased at later stages (from 48 to $120 \mathrm{hpi}$ ) but not in NbSABP2-silenced plants (Fig. 7). As expected, no significant change was observed for MeSA in NbSAMT-silenced plants and $N a h G$-transgenic plants. Most interestingly, we found that the amounts of released MeSA and SA were significantly reduced when JA and MeJA are reduced in NbOPR3- and NbJMT-silenced plants compared with the control plants (Figs. 6 and 7). These results indicate that JA signaling could regulate $\mathrm{SA}$ and MeSA production.

In order to further corroborate the results of phytohormone analyses indicating that JA signaling could regulate SA and MeSA production, the expression of SA and JA biosynthetic genes were examined upon two kinds of hormone applications.
NbICS1 (SA biosynthetic gene) and NbOPR3 (JA biosynthetic gene) were upregulated upon SA and JA treatment, respectively (Supplementary Fig. S7). Interestingly, the expression of NbICS1 was also upregulated upon JA application in comparison with basal expression level. In contrast, the expression of NbOPR3 was not induced upon SA treatment. These results further indicate that JA signaling could regulate SA and MeSA production.

Taken together, our results suggest that JA and MeJA first accumulate in phloem exudates of leaves challenged with TMV at early stages and MeSA accumulates at later stages. Consistent with their temporal accumulations, JA and MeJA accumulations at early stages are required for MeSA and SA production at later stages after TMV infection in $N$. benthamiana plants.

\section{DISCUSSION}

Both SA and JA are important components of the complex signaling networks that mediate plant defense responses against pathogens and are implicated in systemic resistance responses. However, the nature of the mobile signal for establishing systemic resistance remains elusive (Vlot et al. 2008a). Moreover, the mechanisms for positive interaction between JA and SA are not known. In this report, we have shown that both MeSA
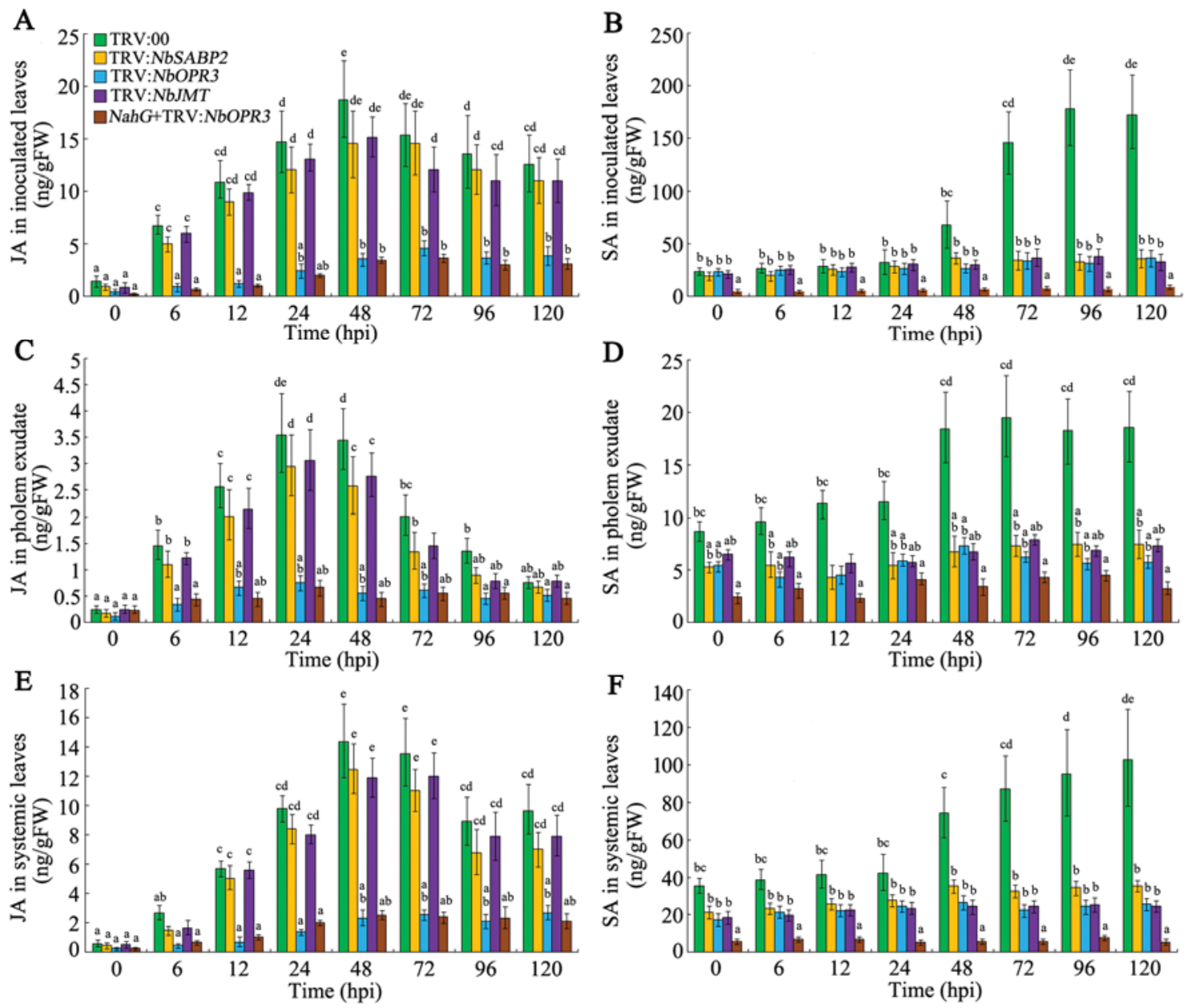

Fig. 6. Jasmonic acid (JA) and salicylic acid (SA) levels in the gene-silenced plants infected with TMV-green fluorescent protein (GFP) from 0 to $120 \mathrm{~h}$ postinfection (hpi). A, JA and B, SA levels in the inoculated leaves of gene-silenced plants infected with TMV-GFP from 0 to 120 hpi. C, JA and D, SA levels in phloem exudate of gene-silenced plants infected with TMV-GFP from 0 to $120 \mathrm{hpi}$. E, JA and $\mathbf{F}$, SA levels in systemic leaves of gene-silenced plants infected with TMV-GFP from 0 to 120 hpi. The assay was performed on NbSABP2-, NbOPR3-, and NbJMT-silenced and control (TRV:00) Nicotiana benthamiana seedlings and NbOPR3-silenced NahG-transgenic plants. Bars represent mean and standard deviation of values obtained from three biological replicates per genotype and time point. Different lowercase letters indicate significant differences $(P<0.05)$. 
and jasmonate signaling are essential for systemic resistance against TMV in $N$. benthamiana plants. Our results also show that foliar application of JA followed by SA activates the strongest systemic defense response and upregulates the expression of defense-related genes against TMV-GFP in $N$. benthamiana plants, which is consistent with a recent study showing that application of JA and SA could improve plant resistance to RNA viruses (Shang et al. 2011). We also showed that JA or MeJA accumulates at early stages and SA or MeSA at later stages of TMV infection and JA or MeJA accumulation is required for SA or MeSA accumulation, providing a mechanism for the interaction between these two hormones in systemic resistance response against TMV.

Using an efficient VIGS, we found that silencing of SA or JA biosynthetic and signaling genes impaired plant systemic resistance to TMV. Silencing of SA biosynthetic and signaling genes, including NbICS1, NbSABP2, NbSAMT, and NbNPRl, in $N$. benthamiana plants strongly increased susceptibility to TMV-GFP (Fig. 2). Our data support the conclusion that SA plays an important role in systemic resistance and MeSA serves as a long-distance phloem-mobile signal in N. benthamiana plants.

The earliest candidate for a mobile long-distance signal traveling from inoculated to systemic tissue was SA (Yalpani et al. 1991). Tobacco and Arabidopsis plants that are SA deficient or unable to accumulate SA after infection fail to develop SAR and display enhanced susceptibility to pathogen infection (Dong 2001; Kunkel and Brooks 2002). Exogenous SA application increases whole-plant resistance in many species (Kiefer and Slusarenko 2003). However, evidence from detailed physiological and grafting experiments has essentially excluded a function of SA as the phloem-mobile long-distance signal (Attaran et al. 2009; Rasmussen et al. 1991; Vernooij et al. 1994). Instead of SA itself, recent study demonstrates that MeSA or SA glucoside are SAR signals in tobacco and accumulate in phloem exudates in an SABP2-dependent manner (Park et al. 2007, 2009). Recently, Vlot and associates (2008b) characterized a new family of Arabidopsis genes encoding $18 \alpha / \beta$-fold hydrolases that are functionally homologous to NtSABP2 and suggest that MeSA functions as a conserved SAR signal in Arabidopsis. Furthermore, the potato ortholog of tobacco SABP2 (StMES1) has been characterized, which supports the idea that MeSA functions as a conserved SAR signal in potato and possibly in other species (Manosalva et al. 2010; Vlot et al. 2008b).

In addition to SA or MeSA, we found that silencing of JA biosynthetic and signaling genes, including $N b O P R 3, N b J M T$, and $\mathrm{NbCOII}$, also impaired plant systemic resistance (Fig. 2). These data suggested that JA plays a role in SAR against viruses as well. Extensive evidence, particularly from studies in Arabidopsis, suggests the involvement of a lipid or lipid-derived mo-
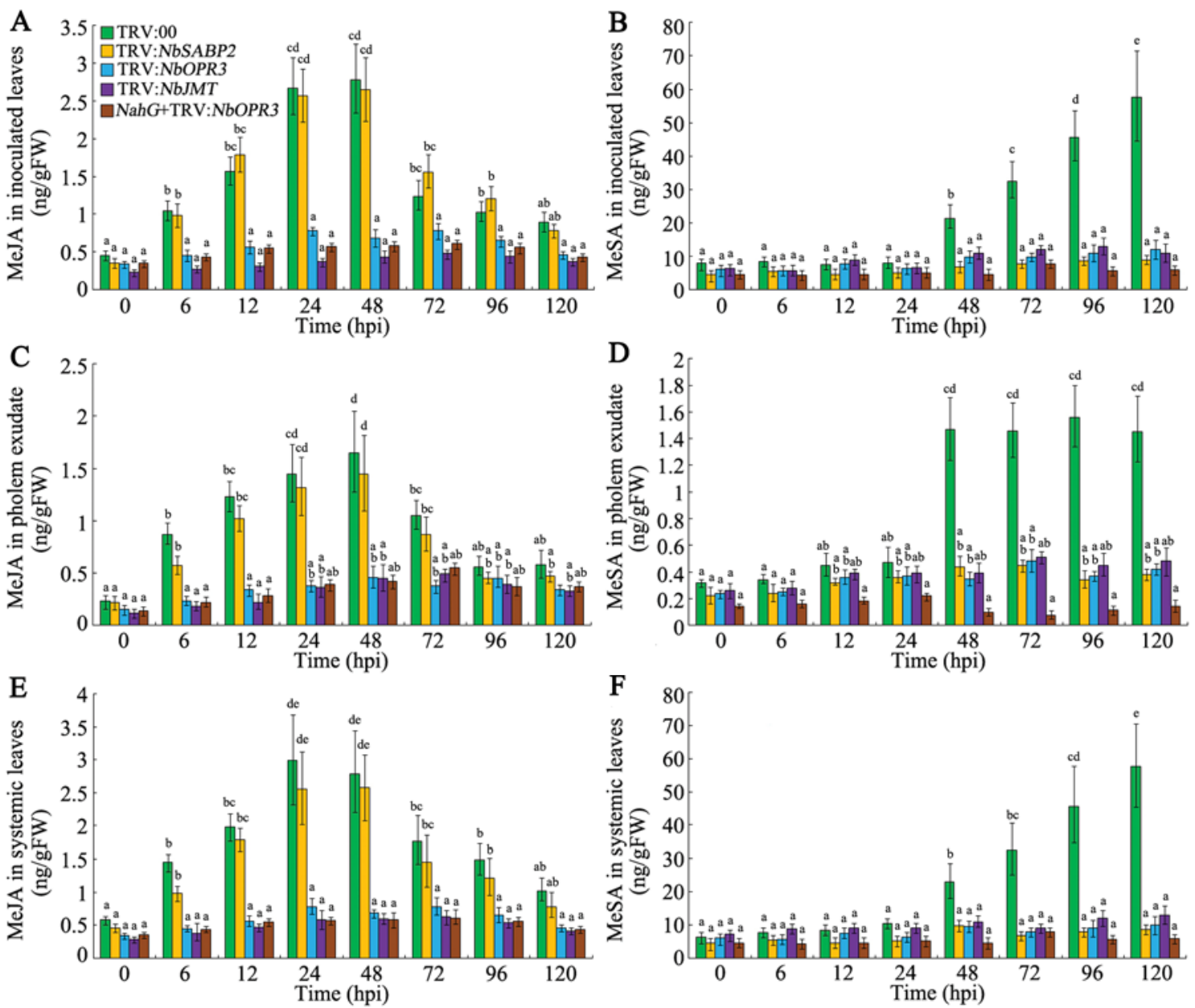

Fig. 7. Methyl jasmonate (MeJA) and methyl salicylate (MeSA) levels in the gene-silenced plants infected with TMV-green fluorescent protein (GFP) from 0 to $120 \mathrm{~h}$ postinfection (hpi). A, MeJA and B, MeSA levels in the inoculated leaves of gene-silenced plants infected with TMV-GFP from 0 to 120 hpi. C, MeJA and D, MeSA levels in phloem exudate of gene-silenced plants infected with TMV-GFP from 0 to 120 hpi. E, MeJA and F, MeSA levels in systemic leaves of gene-silenced plants infected with TMV-GFP from 0 to 120 hpi. The assay was performed on $N b S A B P 2-, N b O P R 3-$, and $N b J M T$-silenced and control (TRV:00) Nicotiana benthamiana seedlings and NbOPR3-silenced NahG-transgenic plants. Bars represent mean and standard deviation of values obtained from three biological replicates per genotype and time point. Significant differences $(P<0.05)$ are denoted by different lowercase letters. 
bile SAR signal (Chaturvedi et al. 2008; Grant and Lamb 2006; Jung et al. 2009; Maldonado et al. 2002; Truman et al. 2007). Mutations in suppressor of fatty acid desaturase 1 encoding dihydroxyacetone phosphate reductase compromise the activation of SAR, suggesting the involvement of plastidic glycerolipids in SAR (Chaturvedi et al. 2008; Nandi et al. 2004). The importance of plant lipid transfer proteins as components in systemic signaling has also been demonstrated (Buhot et al. 2004; Maldonado et al. 2002). Cohen and associates (1993) showed that treatment of potato and tomato with JA led to local and systemic protection against Phytophthora infestans. JA, which plays an important role in defenses against necrotrophic pathogens and insect attack, has also been implicated in SAR signaling. Truman and associates (2007) showed that the JA-signaling mutants suppressor of $g 2$ allele of SKP1 $1 b$, opr3, and jasmonate insensitive 1 failed to develop SAR upon leaf infiltration with Pseudomonas syringae pv. tomato DC3000. JA but not SA accumulates in petiole exudates, which is accompanied by the induction of JA biosynthesis genes in systemic leaves challenged with $P$. syringae pv. tomato DC3000 (avrRpml). Our results suggest that JA biosynthetic and signaling genes also play an important role in antagonizing TMV-GFP infection.

Although both JA and MeJA and SA and MeSA are required for systemic resistance responses, they don't seem to act additively or synergistically (Fig. 4). Rather, our data suggest that JA, MeJA, SA, and MeSA act sequentially during systemic resistance response after TMV infection. First, in our hormone application experiments, the application of JA followed by SA always induces the best resistance (Fig. 1). Second, the JA marker genes are increased before SA marker genes after TMV infection (Fig. 3). Third, hormone measurements demonstrated that, whereas JA and MeJA are increased during early stages of TMV infection (6 to $48 \mathrm{hpi}$ ), SA and MeSA are increased at later stages (72 to 120 hpi) (Figs. 6 and 7). Finally, blocking JA or MeJA formation greatly reduces the subsequent SA or MeSA accumulation but blocking SA or MeSA has no clear effects on JA or MeJA accumulation (Figs. 6 and 7), suggesting that JA or MeJA act to stimulate SA or MeSA accumulation. Therefore, our results suggest a previously unknown interaction between these two important hormones in systemic response against a virus pathogen.

The evidence for the sequential actions of SA and JA in SAR is in contrast to the general assumption that SA and JA signaling pathways are antagonistic (Bari and Jones 2009). However, synergistic interactions between SA and JA signaling have been reported before (Clarke et al. 2009; Cui et al. 2005; Mishina and Zeier 2007; Mur et al. 2006; van Wees et al. 2000). Recently, Spoel and associates (2007) showed that plants tightly control cross-talk between SA- and JA-dependent defenses. Halim and associates (2009) showed that SA and $\mathrm{JA}$ are both required for PAMP-induced defense responses in potato. JA was suggested to strengthen the MeSA component of SAR signaling (Vlot et al. 2008a and b). Attaran and associates (2009) reported that MeSA production induced by avirulent $P$. syringae partially required JA biosynthesis and depends on COI1- and MYC2-mediated downstream signaling, suggesting that JA signaling could regulate MeSA production. Truman and associates (2007) proposed that the JA and SA phases maybe act in tandem. They thought that the characteristics of rapid JA signaling may represent a previously undetected early initiation phase of systemic resistance, whereas SA contributes to subsequent events in establishment of systemic immunity. In this report, when the plants are subjected to virus invasion, they will trigger JA signaling immediately for establishing the early initiation phase of systemic resistance. After that, JA signaling regulates SA and, subsequently, SA signaling is activated strongly to establish a broad systemic resistance. Our results provide evidence to explore the JA-SA interactions observed in other defense responses.

In summary, we have shown that MeSA and jasmonate signaling are essential for systemic resistance in $N$. benthamiana. We also showed that JA and MeJA signaling likely regulate MeSA and SA production in N. benthamiana plants. Thus, our studies revealed a new mechanism for the interaction between JA and SA in mediating systemic resistance response and provide a framework to further understand the molecular mechanisms of plant defense against virus pathogens.

\section{MATERIALS AND METHODS}

\section{Plant materials and growth conditions.}

The NahG-transgenic $N$. benthamiana plants and WT $N$. benthamiana were grown in a greenhouse at $25^{\circ} \mathrm{C}$ and cycles of $16 \mathrm{~h}$ of light and $8 \mathrm{~h}$ of darkness $\left(100 \mu \mathrm{mol} \mathrm{m} \mathrm{m}^{-2} \mathrm{~s}^{-1}\right)$. Seedlings used in the experiments were 6 to 7 weeks old.

\section{Chemical treatments.}

SA, SA analog BTH, JA, and two other JA biosynthesis inhibitors (SHAM and DIECA) were purchased from Sigma (St. Louis). All hormone and inhibitor solutions were prepared in water containing $0.02 \%$ ( $\mathrm{vol} / \mathrm{vol}$ ) Tween 20 . The chemicals and the concentrations used are as follows: SA $(300 \mu \mathrm{M})$, JA $(100 \mu \mathrm{M})$, BTH $(500 \mu \mathrm{M})$, SHAM $(500 \mu \mathrm{M})$, and DIECA $(200 \mu \mathrm{M})$. Distilled water containing $0.02 \%$ (vol/vol) Tween 20 was used as a control treatment. All experiments were repeated with similar results.

\section{Construction of VIGS vectors.}

Partial cDNA of NbICS1, NbSABP2, NbSAMT, NbNPR1, $N b O P R 3, N b J M T$, and $N b C O I 1$ was amplified by PCR from a cDNA library of $N$. benthamiana leaf tissues using genespecific primers. These gene-specific primers are shown in Supplementary Table S1. The RT-PCR products were cloned into pCR8/GW/TOPO vector from a TOPO TA cloning kit (Invitrogen, Carlsbad, CA, U.S.A.), according to the instructions of the manufacturer. Then, partial fragments of these genes were inserted into the TRV vector (pTRV-RNA2).

\section{TRV-mediated VIGS assay and pathogen inoculation.}

For VIGS assay, pTRV1 or pTRV2 and its derivatives were introduced into Agrobacterium strain GV2260 by electroporation (Bio-Rad, Hercules, CA, U.S.A.). Further details are shown in Supplementary Methods S1.

\section{GFP imaging.}

GFP fluorescence was photographed under UV light using a Canon G11 digital camera and a B-100AP longwave-UV lamp (Ultra-Violet Products, Upland, CA, U.S.A.) (Zhu et al. 2013).

RNA extraction, RT-PCR, and quantitative real-time PCR.

Total RNAs were isolated from $N$. benthamiana leaves as previously described (Xu et al. 2012). The quantitative realtime PCR and RT-PCR analysis was performed with the primers shown in Supplementary Table S2. Further details are shown in Supplementary Methods S2.

\section{Protein extraction and Western blot analysis.}

Total proteins were extracted with extraction buffer $(50 \mathrm{mM}$ Tris-Cl [pH 6.8], 5\% mercaptoethanol, $10 \%$ glycerol, $4 \%$ sodium dodecyl sulfate, and $4 \mathrm{M}$ urea) in an ice bath. Protein concentrations were determined by the Bradford method using bovine serum albumin as a standard (Bradford 1976). Western blot analysis was performed according to Zhu and associates (2012). Further details are shown in Supplementary Methods S3. 


\section{Collection of phloem exudates.}

Leaf exudate was collected as described previously (Park et al. 2007). In brief, the petioles of leaves were cut under $1 \mathrm{mM}$ EGTA (pH 7.0), and placed in 6-ml vials containing $3 \mathrm{ml}$ of EGTA solution. Tubes with excised leaves were incubated in continuous light, $100 \mu \mathrm{mol} / \mathrm{m}^{2} / \mathrm{s}, 100 \%$ relative humidity, $20^{\circ} \mathrm{C}$, inside 10-liter plastic chambers.

\section{Quantification of SA, MeSA, JA, and MeJA levels.}

Phytohormones were quantified by HPLC mass spectrometry from crude plant extracts according to the method of Pan and associates (2010). The 2-hydroxybenzoic acid- $\left({ }^{2} \mathrm{H}_{6}\right)$ was obtained from Sigma-Aldrich, $\left({ }^{2} \mathrm{H}_{4}\right)$-MeSA was obtained from CDN Isotopes (Quebec, Canada), and dihydro-JA and dihydroMeJA were obtained from OlChemim (Olomouc, Czech Republic) as internal standards.

\section{Statistical analysis.}

Values presented are means \pm standard deviation of at least three replicates. Data were subjected to analysis of variance using JMP IN software (version 4; SAS Institute). The significance of each treatment effect was determined by the magnitude of the $F$ value at $P=0.05$. When a significant $F$ value was obtained for treatments, separation of means was accomplished using Fisher's protected least significant difference at $P=0.05$.

\section{ACKNOWLEDGMENTS}

This manuscript was selected as a feature article through the APS-CSPP Cooperative Agreement. We thank S. Whitham (Iowa State University) for providing pTRV vector, NbPDS silencing vector, and TMV-GFP; and H.-S. Guo (Institute of Microbiology, Chinese Academy of Sciences, Beijing) for providing the $N a h G$-transgenic $N$. benthamiana seed. This work was supported by the National Nature Science Foundation of China (91017004, 31070210, 31171835, and 31270290), Doctoral Foundation of the Ministry of Education (20110181110059 and 20120181130008), and Sichuan and Chengdu Nature Science Foundation (2010JQ0080, 11DXYB097JH-027, and 2012JY0078).

\section{LITERATURE CITED}

Achuo, E. A., Audenaert, K., Meziane, H., and Hofte, M. 2004. The salicylic acid dependent defence pathway is effective against different pathogens in tomato and tobacco. Plant Pathol. 53:65-72.

Adie, B., Chico, J. M., Rubio-Somoza, I., and Solano, R. 2007. Modulation of plant defenses by ethylene. J. Plant Growth Regul. 26:160-177.

Anand, A., Uppalapati, S. R., Ryu, C. M., Allen, S. N., Kang, L., Tang, Y. H., and Mysore, K. S. 2008. Salicylic acid and systemic acquired resistance play a role in attenuating crown gall disease caused by Agrobacterium tumefaciens. Plant Physiol. 146:703-715.

Attaran, E., Zeier, T. E., Griebel, T., and Zeier, J. 2009. Methyl salicylate production and jasmonate signaling are not essential for systemic acquired resistance in Arabidopsis. Plant Cell 21:954-971.

Bari, R., and Jones, J. D. G. 2009. Role of plant hormones in plant defence responses. Plant Mol. Biol. 69:473-488.

Bradford, M. N. 1976. A rapid and sensitive method for the quantitation of microgram quantities of protein utilizing the principle of protein-dye binding. Anal. Biochem. 72:248-254.

Breithaupt, C., Kurzbauer, R., Lilie, H., Schaller, A., Strassner, J., Huber, R., Macheroux, P., and Clausen, T. 2006. Crystal structure of 12-oxophytodienoate reductase 3 from tomato: Self-inhibition by dimerization. Proc. Natl. Acad. Sci. U.S.A. 103:14337-14342.

Buhot, N., Gomes, E., Milat, M. L., Ponchet, M., Marion, D., Lequeu, J. Delrot, S., Coutos-Thevenot, P., and Blein, J. P. 2004. Modulation of the biological activity of a tobacco LTP1 by lipid complexation. Mol. Biol. Cell 15:5047-5052.

Cao, H., Glazebrook, J., Clarke, J. D., Volko, S., and Dong, X. 1997. The Arabidopsis NPRI gene that controls systemic acquired resistance encodes a novel protein containing ankyrin repeats. Cell 88:57-63.

Chanda, B., Xia, Y., Mandal, M. K., Yu, K., Sekine, K. T., Gao, Q. M., Selote, D., Hu, Y., Stromberg, A., Navarre, D., Kachroo, A., and Kachroo, P. 2011. Glycerol-3-phosphate is a critical mobile inducer of systemic immunity in plants. Nat. Genet. 43:421-427.
Chaturvedi, R., Krothapalli, K., Makandar, R., Nandi, A., Sparks, A. A Roth, M. R., Welti, R., and Shah, J. 2008. Plastid $\omega 3$-fatty acid desaturase-dependent accumulation of a systemic acquired resistance inducing activity in petiole exudates of Arabidopsis thaliana is independent of jasmonic acid. Plant J. 54:106-117.

Cheong, J. J., and Choi, Y. D. 2003. Methyl jasmonate as a vital substance in plants. Trends Genet. 19:409-413.

Clarke, S. M., Cristescu, S. M., Miersch, O., Harren, F. J. M., Wasternack, C., and Mur, L. A. J. 2009. Jasmonates act with salicylic acid to confer basal thermotolerance in Arabidopsis thaliana. New Phytol. 182:175-187.

Cohen, Y., Gisi, U., and Niderman, T. 1993. Local and systemic protection against Phytophthora infestans induced in potato and tomato plants by jasmonic acid and jasmonic methyl ester. Phytopathology 83:10541062

Cui, J., Bahrami, A. K., Pringle, E. G., Hernandez-Guzman, G., Bender, C L., Pierce, N. E., and Ausubel, F. M. 2005. Pseudomonas syringae manipulates systemic plant defenses against pathogens and herbivores. Proc. Natl. Acad. Sci. U.S.A. 102:1791-1796.

Dangl, J. L., and Jones, J. D. G. 2001. Plant pathogens and integrated defence responses to infection. Nature 411:826-833.

Dong, X. 2001. Genetic dissection of systemic acquired resistance. Curr. Opin. Plant Biol. 4:309-314.

Durrant, W. E., and Dong, X. 2004. Systemic acquired resistance. Annu. Rev. Phytopathol. 42:185-209.

Erickson, F. L., Holzberg, S., Calderon-Urrea, A., Handley, V., Axtell, M., Corr, C., and Baker, B. 1999. The helicase domain of the TMV replicase proteins induces the $N$-mediated defense response in tobacco. Plant J. 18:67-75.

Forouhar, F., Yang, Y., Kumar, D., Chen, Y., Fridman, E., Park, S. W., Chiang, Y. W., Acton, T. B., Montelione, G. T., Pichersky, E., Klessig, D. F., and Tong, L. 2005. Structural and biochemical studies identify tobacco SABP2 as a methyl salicylate esterase and implicate it in plant innate immunity. Proc. Natl. Acad. Sci. U.S.A. 102:1773-1778.

Fu, Z. Q., and Dong, X. 2013. Systemic acquired resistance: Turning local infection into global defense. Annu. Rev. Plant Biol. 64:839-863.

Gaffney, T., Friedrich, L., Vernooij, B., Negrotto, D., Nye, G., Uknes, S. Ward, E., Kessmann, H., and Ryals, J. 1993. Requirement of salicylic acid for the induction of systemic acquired resistance. Science 261:754 756.

Grant, M., and Lamb, C. 2006. Systemic immunity. Curr. Opin. Plant Biol. 9:414-420

Halim, V., Altmann, S., Ellinger, D., Eschen-Lippold, L., Miersch, O., Scheel, D., and Rosahl, S. 2009. PAMP-induced defense responses in potato required both salicylic acid and jasmonic acid. Plant J. 57:230242.

Jones, J.D., and Dangl, J. L. 2006. The plant immune system. Nature 444:323-329.

Jung, H. W., Tschaplinski, T., Wang, L., Glazebrook, J., and Greenberg, J. T. 2009. Priming in systemic plant immunity. Science 324:89-91.

Kachroo, A., and Robin, G. P. 2013. Systemic signaling during plant defense. Curr. Opin. Plant Biol. 16:527-533.

Katsir, L., Schilmiller, A. L., Staswick, P. E., He, S. Y., and Howe, G. A 2008. COI1 is a critical component of a receptor for jasmonate and the bacterial virulence factor coronatine. Proc. Natl. Acad. Sci. U.S.A. 105:7100-7105.

Kiefer, I. W., and Slusarenko, A. J. 2003. The pattern of systemic acquired resistance induction within the Arabidopsis rosette in relation to the pattern of translocation. Plant Physiol. 132:840-847.

Koornneef, A., and Pieterse, C. M. J. 2008. Cross talk in defense signaling. Plant Physiol. 146:839-844.

Kumar, D., and Klessig, D. F. 2003. High-affinity salicylic acid-binding protein 2 is required for plant innate immunity and has salicylic acidstimulated lipase activity. Proc. Natl. Acad. Sci. U.S.A. 100:1610116106.

Kunkel, B. N., and Brooks, D. M. 2002. Cross talk between signaling pathways in pathogen defense. Curr. Opin. Plant Biol. 5:325-331.

Kwon, S. J., Jin, H. C., Lee, S., Nam, M. H., Chung, J. H., Kwon, S. I., Ryu, C. M., and Park, O. K. 2009. GDSL lipase-like 1 regulates systemic resistance associated with ethylene signaling in Arabidopsis. Plant J. 58:235-245.

Liu, G. S., Holub, E. B., Alonso, J. M., Ecker, J. R., and Fobert, P. R. 2005. An Arabidopsis NPR1-like gene, NPR4, is required for disease resistance. Plant J. 41:304-318.

Liu, Y., Schiff, M., and Dinesh-Kumar, S. P. 2002a. Virus-induced gene silencing in tomato. Plant J. 31:777-786.

Liu, Y., Schiff, M., Marathe, R., and Dinesh-Kumar, S. P. 2002b. Tobacco Rar1, EDS1 and NPR1/NIM1 like genes are required for $N$-mediated resistance to Tobacco mosaic virus. Plant J. 30:415-429.

Maldonado, A. M., Cameron, R. K., Doernert, P., Dixon, R. A., and Lamb, C. 2002. A putative lipid transfer protein involved in systemic resis- 
tance signaling in Arabidopsis. Nature 419:399-403.

Manosalva, P. M., Park, S. W., Forouhar, F., Tong, L., Fry, W. E., and Klessig, D. F. 2010. Methyl esterase 1 (StMES1) is required for systemic acquired resistance in potato. Mol. Plant-Microbe Interact. 23:1151-1163.

Memelink, J. 2009. Regulation of gene expression by jasmonate hormones. Phytochemistry 70:1560-1570.

Mishina, T. E., and Zeier, J. 2007. Pathogen-associated molecular pattern recognition rather than development of tissue necrosis contributes to bacterial induction of systemic acquired resistance in Arabidopsis. Plant J. 50:500-513.

Mur, L. A., Kenton, P., Atzorn, R., Miersch, O., and Wasternack, C. 2006. The outcomes of concentration specific interactions between salicylate and jasmonate signaling include synergy, antagonism, and oxidative stress leading to cell death. Plant Physiol. 140:249-262.

Nahar, K., Kyndt, T., Vleesschauwer, D. D., Höfte, M., and Gheysen, G. 2011. The jasmonate pathway is a key player in systemically induced defense against root knot nematodes in rice. Plant Physiol. 157:305316.

Nandi, A., Welti, R., and Shah, J. 2004. The Arabidopsis thaliana dihydroxyacetone phosphate reductase gene SUPPRESSOR OF FATTY ACID DESATURASE DEFICIENCY 1 is required for glycerolipid metabolism and for the activation of systemic acquired resistance. Plant Cell 16:465-477.

Návarová, H., Bernsdorff, F., Döring, A. C., and Zeier, J. 2012. Pipecolic acid, an endogenous mediator of defense amplification and priming, is a critical regulator of inducible plant immunity. Plant Cell 24:5123-5141.

Pan, X. Q., Welti, R., and Wang, X. M. 2010. Quantitative analysis of major plant hormones in crude plant extracts by high-performance liquid chromatography-mass spectrometry. Nat. Protoc. 5:986-992.

Park, S. W., Kaimoyo, E., Kumar, D., Mosher, S., and Klessig, D. F. 2007. Methyl salicylate is a critical mobile signal for plant systemic acquired resistance. Science 318:113-116.

Park, S. W., Liu, P. P., Forouhar, F., Vlot, A. C., Tong, L., Tietjen, K., and Klessig, D. F. 2009. Use of a synthetic salicylic acid analog to investigate the roles of methyl salicylate and its esterases in plant disease resistance. J. Biol. Chem. 284:7307-7317.

Pieterse, C. M., Van Wees, S. C., Van Pelt, J. A., Knoester, M., Laan, R., Gerrits, H., Weisbeek, P. J., and Van Loon, L. C. 1998. A novel signaling pathway controlling induced systemic resistance in Arabidopsis. Plant Cell 10:1571-1580.

Rasmussen, J. B., Hammerschmidt, R., and Zook, M. N. 1991. Systemic induction of salicylic acid accumulation in cucumber after inoculation with pseudomonas syringae pv. syringae. Plant Physiol. 97:1342-1347.

Rojas, C. M., Senthil-Kumar, M., Wang, K., Ryu, C. M., Kaundal, A., and Mysore, K. S. 2012. Glycolate oxidase modulates reactive oxygen species-mediated signal transduction during nonhost resistance in Nicotiana benthamiana and Arabidopsis. Plant Cell 24:336-352.

Seo, H. S., Song, J. T., Cheong, J. J., Lee, Y. H., Lee, Y. W., Hwang, I., Lee J. S., and Choi, Y. D. 2001. Jasmonic acid carboxyl methyltransferase: A key enzyme for jasmonate-regulated plant responses. Proc. Natl. Acad. Sci. U.S.A. 98:4788-4793.

Shah, J. 2009. Plants under attack: Systemic signals in defence. Curr. Opin. Plant Biol. 12:459-464.

Shah, J., and Zeier, J. 2013. Long-distance communication and signal amplification in systemic acquired resistance. Front. Plant Sci. 4:30.

Shang, J., Xi, D. H., Xu, F., Wang, S. D., Cao, S., Xu, M. Y., Zhao P. P., Wang, J. H., Jia, S. D., Zhang, Z. W., Yuan, S., and Lin, H. H. 2011. A broad spectrum, efficient and nontransgenic approach to control plant viruses by application of salicylic acid and jasmonic acid. Planta 233:299-308
Spoel, S. H., Johnson, J. S., and Dong, X. 2007. Regulation of tradeoffs between plants defenses against pathogens with different lifestyles. Proc. Natl. Acad. Sci. U.S.A. 104:18842-18847.

Truman, W., Bennett, M., Kubigsteltig, I., Turnbull, C., and Grant, M. 2007. Arabidopsis systemic immunity uses conserved defense signaling pathways and is mediated by jasmonates. Proc. Natl. Acad. Sci. U.S.A. 104:1075-1080.

van Wees, S. C. M., de Swart, E. A. M., van Pelt, J. A., van Loon, L. C., and Pieterse, C. M. J. 2000. Enhancement of induced disease resistance by simultaneous activation of salicylate- and jasmonate-dependent defense pathways in Arabidopsis thaliana. Proc. Natl. Acad. Sci. U.S.A. 97:8711-8716.

Vernooij, B., Friedrich, L., Morse, A., Reist, R., Kolditzjawhar, R., Ward, E., Uknes, S., Kessmann, H., and Ryals, J. 1994. Salicylic acid is not the translocated signal responsible for inducing systemic acquired resistance but is required in signal transduction. Plant Cell 6:959-965.

Vlot, A. C., Klessig, D. F., and Park, S. W. 2008a. Systemic acquired resistance: The elusive signal(s). Curr. Opin. Plant Biol. 11:436-442.

Vlot, A. C., Liu, P. P., Cameron, R. K., Park, S. W., Yang, Y., Kumar, D., Zhou, F., Padukkavidanam T., Gustafsson, C., Pichersky, E., and Klessig, D. F. 2008b. Identification of likely orthologs of tobacco salicylic acid-binding protein 2 and their role in systemic acquired resistance in Arabidopsis thaliana. Plant J. 56:445-456.

Wasternack, C. 2007. Jasmonates: An update on biosynthesis, signal transduction and action in plant stress response, growth and development. Ann. Bot. 100:681-697.

Wildermuth, M. C., Dewdney, J., Wu, G., and Ausubel, F. M. 2001. Isochorismate synthase is required to synthesize salicylic acid for plant defence. Nature 414:562-565.

Wu, K., Zhang, L., Zhou, C., Yu, C. W., and Chaikam, V. 2008. HDA6 is required for jasmonate response, senescence and flowering in Arabidopsis. J. Exp. Bot. 59:225-234.

Xie, D. X., Feys, B. F., James, S., Nieto-Rostro, M., and Turner, J. G. 1998. COI1: An Arabidopsis gene required for jasmonate-regulated defense and fertility. Science 280:1091-1094

Xu, F., Yuan, S., Zhang, D. W., Lv, X., and Lin, H. H. 2012. The role of alternative oxidase in tomato fruit ripening and its regulatory interaction with ethylene. J. Exp. Bot. 63:5705-5716.

Yalpani, N., Silverman, P., Wilson, T. M. A., Kleier, D. A., and Raskin, I. 1991. Salicylic acid is a systemic signal and an inducer of pathogenesis-related proteins in virus-infected tobacco. Plant Cell 3:809818.

Zhu, F., Xu, M. Y., Wang, S. D., Jia, S. D., Zhang, P., Lin, H. H., and Xi, D. H. 2012. Prokaryotic expression of pathogenesis related protein 1 gene from Nicotiana benthamiana: Antifungal activity and preparation of its polyclonal antibody. Biotechnol. Lett. 34:919-924.

Zhu, F., Zhang, P., Meng, Y. F., Xu, F., Zhang, D. W., Cheng, J., Lin, H. H., and Xi, D. H. 2013. Alpha-momorcharin, a RIP produced by bitter melon, enhances defense response in tobacco plants against diverse plant viruses and shows antifungal activity in vitro. Planta 237:77-88.

Zhu F., Xi, D. H., Deng, X. G., Peng, X. J., Tang, H., Chen, Y. J., Jian, W., Feng, H., and Lin, H. H. 2014. The Chilli veinal mottle virus regulates expression of the Tobacco mosaic virus resistance gene $N$ and jasmonic acid/ethylene signaling is essential for systemic resistance against Chilli veinal mottle virus in tobacco. Plant Mol. Biol. Rep. 32:32:382-394

\section{AUTHOR-RECOMMENDED INTERNET RESOURCE}

SAS Institute website: www.sas.com 